\title{
PROPERTIES OF AN ABSTRACT PSEUDORESOLVENT AND WELL-POSEDNESS OF THE DEGENERATE CAUCHY PROBLEM
}

\author{
IRINA V. MELNIKOVA \\ Department of Mathematics, Ural State University \\ Pr. Lenina 51, 620083 Ekaterinburg, Russia
}

\begin{abstract}
The degenerate Cauchy problem in a Banach space is studied on the basis of properties of an abstract analytical function, satisfying the Hilbert identity, and a related pair of operators $A, B$.
\end{abstract}

1. We consider in a Banach space $X$ an operator-valued function of complex variable $R(\lambda) \in \mathcal{B}(X)$, satisfying the Hilbert identity:

$$
\forall x \in X \quad R(\lambda) R(\mu) x=\frac{R(\mu)-R(\lambda)}{\lambda-\mu} x, \quad \lambda, \mu \in \Omega \subset \mathbb{C} .
$$

For such function $\operatorname{ker} R(\lambda)=: \mathcal{K}$ and range $R(\lambda)=: \mathcal{R}$ do not depend on $\lambda[1]$. If $\mathcal{K}=\{0\}$, then the function $R(\lambda)$ is called by resolvent, if $\mathcal{K} \neq\{0\}$ - pseudoresolvent. For the case when $R(\lambda)$ is resolvent, from (1) the equality follows:

$$
\lambda-R^{-1}(\lambda)=\mu-R^{-1}(\mu)=: A,
$$

$D(A)=\mathcal{R}$ and $R(\lambda)=R_{A}(\lambda)$.

Let $V(t)$ be an exponentially bounded operator-function $\left(\|V(t)\| \leq L e^{\omega t}\right)$ and

$$
r_{n}(\lambda):=\int_{0}^{\infty} \lambda^{n} e^{-\lambda t} V(t) d t
$$

(the integral exists in the Bochner sense). In [2] it was proved, that $r_{0}(\lambda)$ satisfies (1) for $\operatorname{Re} \lambda>\omega$, iff $V(t)$ satisfies the semigroup relation:

$$
V(t+s)=V(t) V(s), \quad t, s \geq 0,
$$

$r_{n}(\lambda), n \in \mathbb{N}$, satisfies (1); iff

$$
\frac{1}{(n-1) !} \int_{0}^{s}\left[(s-r)^{n-1} V(t+r)-(t+s-r)^{n-1} V(r)\right] d r=
$$

1991 Mathematics Subject Classification: Primary 46C20; Secondary 32G81.

The paper is in final form and no version of it will be published elsewhere. 


$$
=V(t) v(s), \quad s, t \geq 0 ; V(0)=0 .
$$

Relation (4) is taken as the basis in the definition of continuous semigroups (see, for example, [1], [3], [4]), relation (V1) - in the definition of $n$-times integrated semigroups [2].

Definition 1. A one-parameter family of bounded operators $\{U(t), t \geq 0\}$ is called strongly continuous semigroup (or $C_{0}$-semigroup) if the following conditions hold:

(U1) $U(t+h)=U(t) U(h), \quad t, h \geq 0$;

(U2) $U(0)=f$;

(U3) $U(t)$ is strongly continuous with respect to $t \geq 0$.

Definition 2. Let $n \in \mathbb{N}$. A one-parameter family of bounded linear operators $\{V(t), t \geq 0\}$ is called $n$-times integrated exponentially bounded semigroup if (V1) and the following conditions hold

(V2) $V(t)$ is strongly continuous with respect to $t \geq 0$;

(V3) $\exists K>0, \omega \in \mathbb{R}:\|V(t)\| \leq \lambda \exp (\omega t), t \geq 0$.

Definition 3. Semigroup $\{V(t), t \geq 0\}$ is called nondegenerate if

(V4) $\forall t \geq 0 V(t) x=0 \Longrightarrow x=0$.

A $C_{0}$-semigroup is called 0-times integrated semigroup. Operator $A=\lambda-R^{-1}(\lambda)$, $D(A)=\mathcal{R}$ is called the generator of semigroup.

So, an exponentially bounded semigroup by the Laplace transform definies the function $R(\lambda)$, satisfying to (1) and conditions:

$$
\exists l>0, \omega \in \mathbb{R}:|| \frac{d^{k}}{d \lambda^{k}}\left(\frac{R(\lambda)}{\lambda^{n}}\right)|| \leq \frac{L k !}{(\lambda-\omega)^{k+1}}, \quad k=0,1, \ldots
$$

(For case $n=0(5)$ are the conditions of Miyadera-Feller-Phillips-Hille-Yosida, or MFPHY-conditions).

2. Arendt [2] extended on arbitrary Banach space $X$ the abstract criterion for Laplace transform.

Theorem (Arendt-Widder). Let $n \in\{0\} \cup \mathbb{N}, R(\lambda):(\omega, \infty) \rightarrow X$. The condition (5) is equivalent to existence of a function $V(t):[0, \infty) \rightarrow X$, satisfying $V(0)=0$, and

$$
\lim _{\delta \rightarrow 0} \sup _{h \leq \delta} h^{-1}\|V(t+h)-V(t)\| \leq L e^{\omega t}, \quad t \geq 0,
$$

such, that

$$
R(\lambda)=\int_{0}^{\infty} \lambda^{n+1} e^{-\lambda t} V(t) d t
$$

Moreover, $R(\lambda)$ has an analytic extention to $\{\lambda \in \mathbb{C}: \operatorname{Re} \lambda>\omega\}$, which is given by (7).

By virtue of this theorem and results on the connection between well-posedness of the Cauchy problem

$$
\frac{d u(t)}{d t}=A u(t), t \geq 0, u(0)=x,
$$

and existence of semigroup (see, for example [1-4]) we have 
Theorem 1. Let $n \in\{0\} \cup \mathbb{N}, A \in \mathcal{L}(X<X)$. The following statements are equivalent:

(I) For $R(\lambda)=R_{A}(\lambda)$ condition (5) is fulfilled;

(II) $A$ is the generator of $(n+1)$-times integrated semigroup with property $(6)$; that

(III) $(C P)$ is $(n+1)$-well-posed, that is: for any $x \in D\left(A^{n+2}\right)$ unique solution, such

$$
\|u(t)\| \leq L e^{\omega t}\|x\|_{n+1}, \quad\|x\|_{n+1}=\sum_{i=0}^{n+1}\left\|A^{i} x\right\|
$$

exists. If $\overline{D(A)}=X$, then $(\mathrm{CP}) n$-well-posed.

Existence of a resolvent in $\Omega=\{\lambda \in \mathbb{C}: \operatorname{Re} \lambda>\omega,|\operatorname{Im} \lambda|<L \exp (r / n \operatorname{Re} \lambda)\}$ and fulfilment in $\Omega$ of the estimates (MFPHY)-type are equivalent to existence of a local $n$-times integrated semigroup $\{V(t), 0 \leq t<T\}, T>r$ [5], [6]. If for $R(\lambda)>\omega$ only operator $(A-\lambda)^{-1} C$, called by $C$-resolvent $(C \in \mathcal{B}(X)$ is an invertible operator) satisfies to conditions:

$$
\left\|(\lambda-A)^{-k} C\right\| \leq L(R(\lambda)-\omega)^{-k}, \quad k=0,1, \ldots,
$$

then $C$-semigroup with operator $A$ exists [7], the Cauchy problem (CP) is only $C$-wellposed and for such $(\mathrm{CP})$ regularizator, connected with $C$-semigroups, may be constructed $[8]$.

3. Let now $R(\lambda)$ be a pseudoresolvent, such that $\|\lambda R(\lambda)\|$ is bounded. Then like $[1$, section VIII], the following proposition on construction of ker $R$ and range $R$ may be proved.

Proposition 1. For any $x \in X_{1}:=\bar{R}, \lambda R(\lambda) x \rightarrow_{\lambda \rightarrow \infty} x, \mathcal{K} \cap X_{1}=\{0\}, X_{1} \oplus \mathcal{K}=$ $\overline{X_{1} \oplus \mathcal{K}}$ is the subspace in $X$. For a reflexive space $X, X=X_{1} \oplus \mathcal{K}$.

If $R(\lambda)$ is a pseudoresolvent with MFPHY-conditions, then by Arendt-Widder theorem it generates 1-time integrated (degenerate) semigroup $V(t)$ with property $(6)$, such that

$$
R(\lambda)=\int_{0}^{\infty} \lambda e^{-\lambda t} V(t) d t
$$

For $x \in F:=\left\{x \in X: V(t) x \in C^{1}([0, \infty), X)\right\}$ we have

$$
R(\lambda) x=\int_{0}^{\infty} e^{-\lambda t} V^{\prime}(t) x d t,
$$

hence $U(t) x:=V^{\prime}(t) x, x \in F$, satisfies (U1). Due to (6) set $F$ is closed, by definition $F$ $U(t)$ is strongly continuous on $F$, we call it by degenerate $C_{0}$-semigroup. From semigroup property (U1) we have the projector

$$
P:=U^{2}(0)=U(0): F \rightarrow Q:=\operatorname{range} U(0)
$$

and the decomposition of $F$ into the direct sum:

$$
F=Q \oplus \operatorname{ker}(U(0))=Q \oplus \mathcal{K} .
$$

By Definition $1 \tilde{U}(t)=\left.U(t)\right|_{Q}$ is $C_{0}$-semigroup with the generator $G$ :

$$
G x=\lim _{t \rightarrow 0} \frac{\tilde{U}(t) x-x}{t}, \quad x \in D(G), \quad \overline{D(G)}=Q .
$$


4. For the case of a pseudoresolvent, as distinct from the case when $R^{-1}(\lambda)$ exists, we can't connect $G$ with operator, defined by (2) and the Cauchy problem (CP). We show, that $R(\lambda)$ is a pseudoresolvent with MFPHY-conditions, then for any pair of operators $B, A: X \rightarrow E, E-$ a Banach space, such that $B \in \mathcal{B}(X, E), B$ is invertible on $X_{1}$ and $A x=B G x, x \in D(G)$, the degenerate Cauchy problem

$(D C P)$

$$
B \frac{d u(t)}{d t}=A u(t), \quad t \geq 0, \quad u(0)=x, \quad \operatorname{ker} B \neq=\{0\},
$$

is well-posed on $\mathcal{R}$.

Definition 4. Let $A, B \in \mathcal{L}(X, E)$. (DCP) is called uniformly well-posed on $D \subseteq X$, if for any $x \in D$ a solution exists, is unique and

$$
\forall T>0 \exists L>0: \sup _{t \in[0, T]}\|u(t)\| \leq L\|x\| .
$$

It is easily seen that $D \subseteq \mathcal{M}=\{x \in D(A): A x \in$ range $B\}$.

Proposition 2. Let $A, B \in \mathcal{L}(X, E)$ such, that operator $(\lambda B-A)^{-1} B$ is bounded, $\lambda \in \Omega \subset C$. Then $(\lambda B-A)^{-1} B$ satisfies the Hilbert identity and

$$
\mathcal{R}=\operatorname{range}(\lambda B-A)^{-1} B=\mathcal{M} .
$$

Pr o of. The proof of the resolvent identity is routine. We show $\mathcal{R}=\mathcal{M}$. Let $x \in \mathcal{R}$, then $x=(\lambda B-A)^{-1} B y, y \in X$, hence $A x=B(\lambda x-y)$, and $x \in \mathcal{M}$. Conversely, if $A x=B y$ for some $y \in X$, then $(\lambda B-A) x=B(\lambda x-y)$ and $x \in \mathcal{R}$.

Definition 5. Let $X, E$ are Banach spaces. $A, B \in \mathcal{L}(X, E)$ are called generators of degenerate $n$-times integrated semigroup $\{V(t), t \geq 0\} \in \mathcal{B}(X, X)$ if $A$ is closed, $B$ bounded and

$$
R_{A, B}(\lambda):=(\lambda B-A)^{-1} B=\int_{0}^{\infty} \lambda^{n} e^{-\lambda t} V(t) d t, \quad \operatorname{Re} \lambda>\omega .
$$

Theorem 2. Let $A, B$ be generators of degenerate (1-time) integrated semigroup $V(t)$, satisfying (6), then

$$
\begin{gathered}
R_{A, B}(\lambda) V(t)=V(t) R_{A, B}(\lambda), \quad t \geq 0, \operatorname{Re} \lambda>\omega ; \\
t B x=B V(t) x-A \int_{0}^{t} V(s) x d s, \quad x \in X_{1} \oplus \mathcal{K}, \mathcal{K}=\operatorname{ker} B ; \\
B \frac{d}{d t} V^{\prime}(t) x=A V^{\prime}(t) x, \quad x \in R(\lambda)\left(X_{1}\right) .
\end{gathered}
$$

$V^{\prime}(t)$ is degenerate $C_{0}$-semigroup on $F=X_{1} \oplus \mathcal{K}$.

Proof. Let $\lambda, \mu>\omega$, as $R(\lambda)=R_{A, B}(\lambda)$ is a pseudoresolvent, for any $x \in X$

$$
\begin{aligned}
& \int_{0}^{\infty} \mu e^{-\mu t} V(t) R(\lambda) x d t=R(\mu) R(\lambda) x= \\
& =R(\lambda) R(\mu) x=\int_{0}^{\infty} \mu e^{-\mu t} R(\lambda) V(t) x d t
\end{aligned}
$$

and hence by uniqueness of the Laplace transform we have (11). 
Let $x \in X, \operatorname{Re} \lambda>\omega$, then

$$
\begin{aligned}
& \int_{0}^{\infty} \lambda^{2} e^{-\lambda t} t B x d t=B x=(\lambda B-A) R(\lambda) x= \\
& \int_{0}^{\infty} \lambda^{2} e^{-\lambda t} B V(t) x d t-A \int_{0}^{\infty} \lambda e^{-\lambda t} V(t) x d t .
\end{aligned}
$$

Let now $x \in \mathcal{R}, x=R\left(\lambda_{0}\right) y, y \in X$, then

$$
\begin{gathered}
\|A V(t) x\|=\left\|A V(t) R\left(\lambda_{0}\right) y\right\|=\left\|A R\left(\lambda_{0}\right) V(t) y\right\|= \\
=\left\|\lambda_{0} B R(\lambda) V(t) y-B V(t) y\right\| \leq L\|B\| e^{\omega t}\left(\left|\lambda_{0}\|\mid\| x\|+\| y \|\right),\right.
\end{gathered}
$$

that means the Laplace transform of $A V(t) x$ is defined as $A$ is closed we have

and

$$
\int_{0}^{\infty} \lambda^{2} e^{-\lambda t} t B x d t=\int_{0}^{\infty} \lambda^{2} e^{-\lambda t}\left[B V(t) x-\int_{0}^{t} A V(s) x d s\right] d t
$$

$$
t B x=B V(t) x-A \int_{0}^{t} A V(s) x d s, \quad x \in \mathcal{R} .
$$

This equality is true for $x \in \overline{\mathcal{R}}$ and $x \in \operatorname{ker} B$ too, hence we have (12).

It was shown above, that $R(\lambda)$ in (8) satisfies MFPHY-conditions then $U(t)=V^{\prime}(t)$ is degenerate $C_{0}$-semigroup on $F=Q \oplus \mathcal{K}$ and $C_{0}$-semigroup on $Q$. For the generator of such semigroup $D(G)$ is dense in $Q$, hence $F_{1}:=\left\{x \in X: \forall t \geq 0 \exists U^{\prime}(t) x\right\}$ is dense in $Q$ and since $\operatorname{ker} B=\mathcal{K} \subset F_{1}$ we have $\overline{F_{1}}=F$. For $x \in F_{1}$

$$
\lambda R(\lambda) x=\int_{0}^{\infty} \lambda e^{-\lambda t} U(t) x d t=U(0) x+\int_{0}^{\infty} \lambda e^{-\lambda t} U^{\prime}(t) x d t,
$$

and

$$
\lim _{\lambda \rightarrow \infty} \lambda R(\lambda) x=U(0) x
$$

Since operators $\lambda R(\lambda)$ are bounded, (14) is true for $x \in F$. Hence by Proposition 1 $Q \subset X_{1}$. Let $x \in R(\lambda)\left(X_{1}\right)=: \mathcal{R}_{1}, x=R(\lambda) y, y \in X_{1}$, we show (13) and $X_{1} \subset Q$, hence $X_{1}=Q$. For $y \in X_{1}$ (12) is true, we apply operator $(\lambda B-A)^{-1}$ to (12), having used (11) and the equality

$$
(\lambda B-A)^{-1} A y=\lambda(\lambda B-A)^{-1} B y-y, \quad y \in D(A),
$$

we obtain

$$
t x=V(t) x-\int_{0}^{t} V(s)\left(\lambda(\lambda B-A)^{-1} B-I\right) y d s,
$$

hence $x \in F$ and $\mathcal{R}_{1} \subset F$. By Proposition 1 for $x \in \mathcal{R} \lambda R(\lambda) x \rightarrow_{\lambda \rightarrow \infty} x$, hence $\overline{\mathcal{R}_{1}}=X_{1}$ and $X_{1} \subset \bar{F}=F$, as $X_{1} \cap \mathcal{K}=\{0\}, X_{1} \subset Q, F=X_{1} \oplus \mathcal{K}$.

By differentiating (15), applying operator $B$ and differentiating once more we obtain (13).

Theorem 3. Let $A, B \in \mathcal{L}(X, E)$ is closed, $B \in \mathcal{B}(X, E)$ and $R(\lambda)=(\lambda B-A)^{-1} B$ satisfies to MFPHY-conditions, then $(D C P)$ is well-posed on $\mathcal{R}_{1}=R(\lambda)\left(X_{1}\right)$.

Proof. Existence of the solution $u(t)=U(t) x, x \in \mathcal{R}_{1}$, follows from Theorem 2, uniqueness may be proved as in the nondegenerate case (see [3], [4]). 
5. In view of Proposition 2 , set $\mathcal{R}$ coincides with maximal well-posedness class for $(\mathrm{DCP}): \mathcal{R}=\mathcal{M}$. We establish conditions on the pseudoresolvent, connected with operators $A, B$, which assure (DCP) well-posedness on $\mathcal{R}$.

Theorem 4. Let $A, B \in \mathcal{L}(X, E), A$ is closed, $B$ and $R(\lambda)=(\lambda B-A)^{-1} B$ for some $\lambda$ are bounded. Then the following statements are equivalent.

(I) (DCP) is uniformly well-posed on $\mathcal{R}$.

(II) $A, B$ are the generators of a degenerate $C_{0}$-semigroup.

(III) For $R(\lambda)$ MFPHY-conditions are fulfilled and $X=\mathcal{K} \oplus \mathcal{R}$.

Proof. $(\mathrm{I}) \Longrightarrow(\mathrm{II})$. Define on $\mathcal{R}$ operators $\tilde{U}(t)$ as solution operators: for $x \in \mathcal{R}$ $\tilde{U}(t) x:=u(t)$. Similarly to the nondegenerate case the operators $\tilde{U}(t)$ form a semigroup on $X_{1}=\overline{\mathcal{R}}$ and satisfy the equality

$$
(\lambda B-A)^{-1} \int_{0}^{\infty} \lambda^{2} e^{-\lambda t} \tilde{U}(t) x d t=B x, \quad x \in X_{1} .
$$

Operator $(\lambda B-A)$ is invertible for $\operatorname{Re} \lambda>\omega$ : really, let $x \in \operatorname{ker}(\lambda B-A)$, then for $v(t):=\exp (\lambda t) x$ we have

$$
B v^{\prime}(t)=\lambda B v(t)=A v(t), \quad v(0)=x,
$$

and $\|v(t)\|=\|\tilde{U}(t)\| \leq L e^{\omega t}\|x\|, t \geq 0$. On the one hand $\ln \|v(t)\| / t=\operatorname{Re} \lambda+\ln \|x\| / t$, on the other $\ln \|v(t) / t \leq \omega+\ln L\| x \| / t$, and $\operatorname{Re} \lambda \leq \omega$. For generator of $\tilde{U}$ from (16) follows:

$$
(\lambda B-A)^{-1} B x=(\lambda-G)^{-1} x, \quad x \in X_{1}, \quad \operatorname{Re} \lambda>\omega,
$$

operator $P x:=(\lambda-G)\left(\lambda_{0} B-A\right)^{-1} B x, P: X \rightarrow X_{1}$ is projector in $X$ and $U(t):=\tilde{U}(t) P$ is degenerate $C_{0}$-semigroup.

$(\mathrm{II}) \Longrightarrow(\mathrm{III})$. It is not difficult to verify, that $A, B$ are the generators of integrated semigroup $V(t):=\int_{0}^{t} U(t) d t$, satisfying (6). Hence by (8) MFPHY-conditions for $R(\lambda)$ are fulfilled and by Theorem $2 X=F=\mathcal{K} \oplus X_{1}$.

$(\mathrm{III}) \Longrightarrow(\mathrm{I})$. If (III), then $R(\lambda)\left(X_{1}\right)=R(\lambda)(X)=\mathcal{R}$ and by Theorem 3 (I) follows.

In [6] some results on connection between properties of a pseudoresolvent and wellposedness of the differential inclusion: $u^{\prime}(t) \in J u(t), T \geq 0, u(0)=x$, where $J:=B^{-1} A$ with $D(J)=\mathcal{M}$, are obtained by the technique of multivalued operators.

\section{References}

[1] K. Yosida, Functional Analysis, Springer-Verlag, Berlin and New York, 1980.

[2] W. Arendt, Vector valued Laplace transforms and Cauchy problems, Israel J. Math. 59 (1987), 59 pp.

[3] S. G. Krein, Linear differential equations in a Banach space, Amer. Math. Soc., Providence, R.I., 1972.

[4] V. K. Ivanov, I. V. Melnikova, and A. I. Filinkov, Differential-operator equations and ill-posed problems, Nauka, Moscow, 1994 [in Russian].

[5] N. Tanaka and I. Miyadera, Exponentially bounded $C$-semigroups and integrated semigroups, Tokyo J. Math. 12, no. 1 (1989), 99-115. 
[6] I. V. Melnikova and M.A. Alshansky, Well-posedness of the Cauchy problem in a Banach space: regular and degenerate cases, J. Math. Sci., Plenum, 1994, to appear.

[7] E. B. Davies and M. M. Pang, The Cauchy problem and a generalizations of the HilleYosida theorem, Proc. London Math. Soc. 55 (1987), 181-208.

[8] I. V. Melnikova and S.V. Bochkareva, C-semigroups and regularization of an ill-posed Cauchy problem, Russia Acad. Sci. Docl. Math. 47, no. 2 (1993), 228-232. 